\title{
Audio-Visual Entrainment Influence on Postural Dynamics
}

\section{Zlatogor Minchev ${ }^{a, b}$, Emil Kelevedjiev $^{b}$ and Plamen Gatev ${ }^{c}$}

a Institute of Information and Communication Technologies, Bulgarian Academy of Sciences, Acad. G. Bonchev Str., BI. 25A, Sofia 1113, Bulgaria

${ }^{b}$ Institute of Mathematics and Informatics, Acad. G. Bonchev Str., Bl.8, Sofia 1113, Bulgaria

c Institute of Neurobiology, BAS, Acad. G. Bonchev Str., BI. 23, Sofia 1113, Bulgaria

ABSTRACT: The influence of multimedia on modern digital users is constantly growing. The paper describes an experimental study of audio-visual (AV) entrainment towards dynamical changes of selected human biometrics. Special wearable equipment of AV stimulation and polyphysiographic postural and brain activities recordings were accomplished for entrainment effect exploration. The obtained results demonstrated evident quantitative changes in both center-ofpressure sway and EEG of the studied subjects after the entrainment session, outlined with time-frequency spectral and multifractal measures. Practical implementation of the study results is directly applicable to the rehabilitation goals and nowadays digital world people stress relaxation, together with working capacity enlargement by multimedia applications.

KEYWORDS: Audio-visual entrainment, postural dynamics, EEG dynamics, time-frequency spectral analysis, multifractal analysis

\section{Introduction}

The audio-visual entrainment is a wellknown technique for a brain influence and possible locking into the stimulation rhythm dynamics. ${ }^{1}$ It is also used as a neurofeedback therapy approach that could be qualitatively measured from the brain cortex area. ${ }^{2}$

Two key moments have to be noted for this field of study exploration: (i) the implemented stimulation modality and (ii) the stimulation series dynamics.
Both audio and visual modalities with particular applications from the psychological perspective are dating back to the early EEG studying. ${ }^{3}$

Different approaches for the time intervals of the stimulation packets variation (isochronic, monaural or binaural) and spectrum (both audio and visual 'colouring') could be implemented, regarding the desired effect. ${ }^{1,4}$

Another interesting moment of the entrainment application is the possible 
correlation of the brain dynamic changes with other biometric characteristics.

Several studies in this hypothesis support are addressing the similarity of fractal properties between signals reflecting brain, heart and motor activities, $^{5-8}$ but quantitative evidences for their possible joint stimulation cumulative effect are still scarce. $^{9}$

Noting the fact that nowadays multimedia users are relying on wearable entertainment devices, ${ }^{10}$ this could be a quite interesting and valuable applied research direction.

The paper is trying to address the quiet and sensory-conflicted stance and interrelated brain dynamics entrainment, evaluating the resulting cortical and equilibrium changes and their association.

Further on, a methodological approach with a practical follow-up based on quantitative entrainment effect measurements will be considered.

\section{Experimental Framework}

The general experimental framework for AV-entrainment of brain and stance dynamics ${ }^{11}$ is presented in Figure 1.

The experimental framework is encompassing the following key systems:

- Nation ${ }^{\circledR} 7128 \mathrm{~W}$ - C20 wearable, wireless polyphysiographic device, providing 21 channel EEG recording. The EEG activity was monitored and stored in real time on laptop Dell Inspiron 7520 with Nation ${ }^{\circledR}$ specialized EEG software, using sampling frequency $-f_{s}=512 \mathrm{~Hz}$. Six $\mathrm{Ag} / \mathrm{AgCl}$ lead electrodes, following the Jasper recording scheme ${ }^{12}$ of the frontal, central and parietal brain areas were used: Fp1, Fp2, C3, C4, P3 and P4.

The ground electrode was mounted on the forehead and the referencing electrodes 01 \& 02 (averaged) - on the processi mastoidei. Conductive EEG adhesive paste Ten $20^{\odot}$ was used after skin preparation for low noise and reliable recording.

- Tekscan Evolution ${ }^{\circledR}$ low profile pedobarographic platform and HP Probook 6570B laptop with special research software were applied for study of stance dynamics, using center-of-pressure (COP) anterior-posterior sways monitoring and recording before and after the entrainment session.

The AV-entrainment was accomplished with David Delight Plus session stimulator of Mind Alive Inc., Canada, together with white LED nontransparent googles - a Tru-Vu Omniscreen ${ }^{\circledR}$ Eyeset and $\mathrm{Hi}$-Fi stereo headphones.

A "Feeling better: Mood booster-1" mode $^{13}$ (visual stimulation of left sides of visual fields of both eyes, targeting the right cortical hemisphere $-10 \mathrm{~Hz}$ (alpha) and right sides of visual fields of both eyes, targeting the left cortical hemisphere - $18 \mathrm{~Hz}$ (beta)) with 30 minutes duration for alpha and beta frequency bands, respectively, isochronic AV stimulation was used. This pattern of stimulation actually targets settling down negative emotions, relaxing the right, while stimulating the left brain hemisphere to rebalance alpha asymmetry. The visual stimulation was done with eyes-closed and it was accompanied by an audio-stimulation for facilitating visual stimulation.

The experiment was organized in three stages: (i) initial measuring of EEG and COP dynamics before the AV-entrainment session, while standing with eyes-open - EO and eyes-closed - EC (two $1 \mathrm{~min}$ sessions each condition randomly ordered). A sensory conflict due 
to the absence of vision was introduced with EC condition (ii) 30 minutes AV entrainment 'Feeling better: Mood booster $-1^{\prime}$ mode alpha $10 \mathrm{~Hz}$ to beta $18 \mathrm{~Hz}$; (iii) post-entrainment measuring of EEG and COP dynamics with EO and EC immediately after the AV-entrainment session and 10, 20 and $40 \mathrm{~min}$ after the entrainment. The studied healthy experimental subjects were instructed to stand in upright position during (i) and (iii) as quiet as possible.

The AV-entrainment session (ii) was organized in a comfortable sitting position due to its longer duration. Before the experiments all subjects signed standard informed consent. We applied excluding and including criteria sets for psychophysiological studies.

\section{Analysis and Results}

The recorded EEG and COP signals were processed in Matlab R2011b environment. Initial EEG artifacts and outliers removing were performed with 2 SDs accuracy.

Further EEG signal filtering with Butterworth band pass $(0.5-80 \mathrm{~Hz})$ and 12 $\mathrm{dB} /$ Oct, followed by Chebishev $50 \mathrm{~Hz}$ notch - $18 \mathrm{~dB} /$ Oct were used for bandwidth limitation and hum suppression. Due to the high sharpness of the filters, an additive $3 \mathrm{~dB} /$ Oct was stepwise applied.

In order to study the EEG signals two stages were performed: (i) We start with Relative Fourier Power Spectra (RPS) analysis $^{14}$ in four different frequency bands: alpha, theta, beta and gamma.

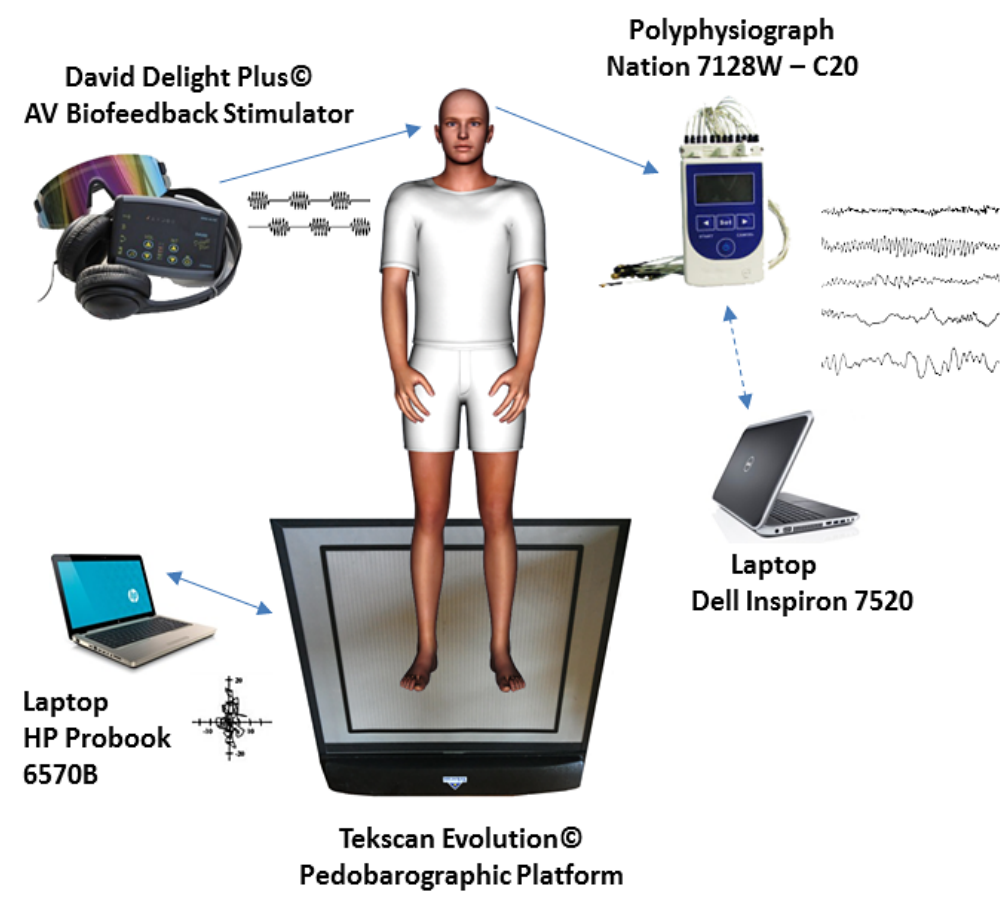

Figure 1: Experimental framework for AV-entrainment of brain and stance dynamics. ${ }^{11}$ 
Zlatogor Minchev, Emil Kelevedjiev and Plamen Gatev
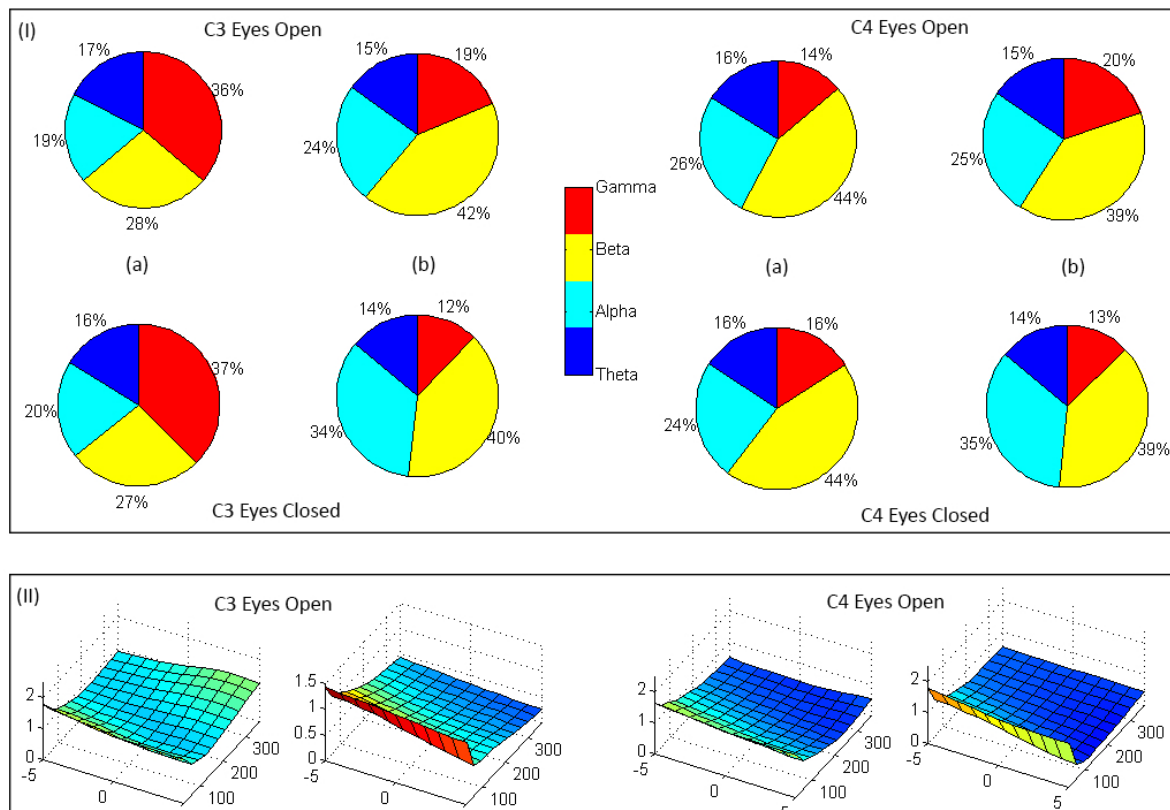

(a)

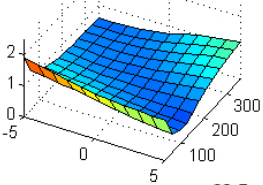

C3 Eyes Close

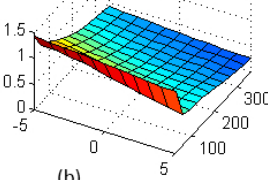

(b)
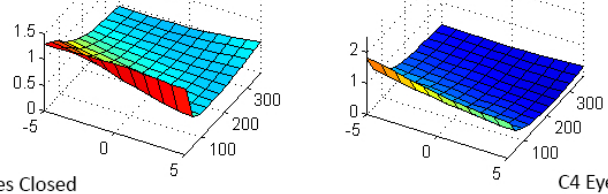

C4 Eyes Closed

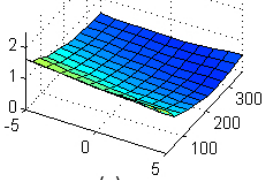

(a)

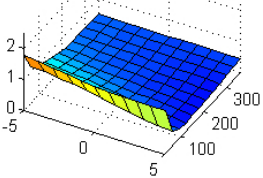

(b)

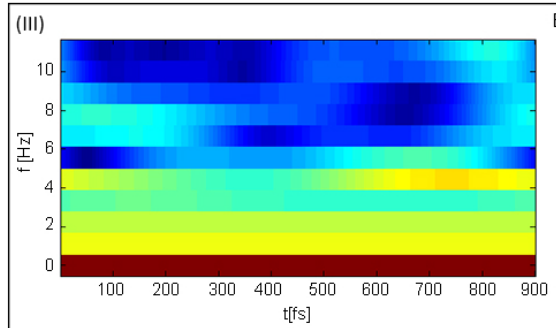

(a)

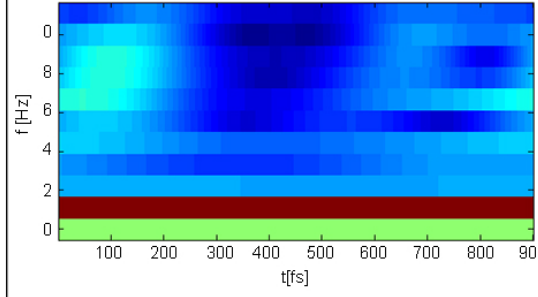

Eyes Open

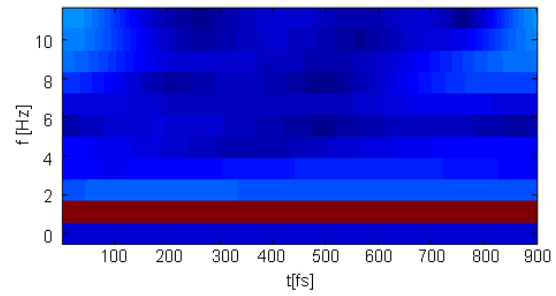

(b)

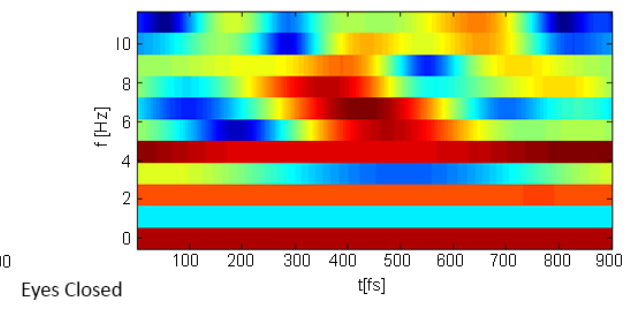

Eyes Closed

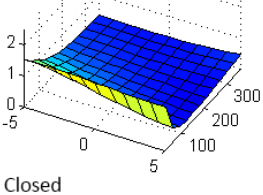

Figure 2: Generalization of the results for: EEG C3 and C4 leads RPS (panel I), MFS (panel II) and COP S-transform dynamics (panel III), before (a) and $10 \mathrm{~min}$ after (b) the $30 \mathrm{~min}$ AV-entrainment session. 
This provides a qualitative EEG evaluation by means of the stimulation in mood booster mode and is rather convenient for initial orientation for such complex signals exploration; (ii) a multifractal spectrum (MFS) calculation based on multiscale multifractal analysis for exploring the Hurst surface ${ }^{6}(h(q, s)$ - dependence of the local Hurst exponent $h$ on the multifractal parameter $q$ and the scale of $s$ observations) and understanding the entrainment EEG scaling effect.

The COP anterior/posterior sway dynamics was analyzed by using S-transform time-frequency analysis. We found it sufficiently illustrative for the AV-entrainment and common from other physiological studies. ${ }^{15}$ Direct observations of the postural sways amplitudes do not produce a clear understanding of the COP dynamics changes, proving the significance of the method described.

The obtained results (see Figure 2) demonstrate a clear contralateral effect (alpha \& beta frequency bands stimulated augmentation with 8-12 \%) of the AV-entrainment for the EEG spectrum, regarding the central area (C3 \& C4 leads) for both EO (normal) and EC (sensory-conflicted) conditions. This is combined with consecutive changes of the EEG multifractal dynamics that clearly is demonstrating a transformation by means of Hurst surface variability diminution.

Additionally, the COP anterior/ posterior dynamics was also correlated with the EEG changes during the non-sensory conflicted measurement - EO condition. The conflicted one showed the negative effect of the session - "disentrainment" regarding the broader S-transform spectrum of the stance.
The postural anterior-posterior sways amplitudes do not showed COP dynamics changes between series EO and EC as well between before and after series.

\section{Discussion}

Obviously, the obtained results are outlining both positive and negative effects of the AV-entrainment.

More concrete, the positive effects could be implemented in the rehabilitation area, working capacity augmentation, stress relaxation or different brain and stance disorders treatment.

The negative effects are opening the vast field of application, concerning the modern multimedia influence and the wearable web technologies and services progress, together with the human factor response.

This is also related and to the emotions and behavioural changes in the human lifestyle, which in the digital world could become problematic due to the increased technological impact.

\section{Acknowledgements}

This study is financially supported by $\mathrm{Na}$ tional Science Fund, Ministry of Education \& Science, Republic of Bulgaria, projects: (i) Cortical Regulation of the Quiet Stance during Sensory Conflict, TK 02/60, www.cleverstance.com; A Study on IT Threats and Users Behaviour Dynamics in Online Social Networks, DMU03/22, Young Scientists Grant, www.facebook.com/snfactor.

\section{References}

1. Siever D. Audio-visual entrainment: history and physiological mechanisms. Association for applied psychophysiology and biofeedback. Biofeed- 
back magazine. 2003 summer; 31(2), [cited 2015 March 10]. Available from: https://goo.gl/EDwhN2.

2. Evans J, editor. Handbook of neurofeedback (dynamics \& clinical applications). New York: The Haworth Medical Press; 2007.

3. Huang T \& Charyton Ch. A comprehensive review of the psychological effects of brainwave entrainment. Altern Ther Health Med. 2008 Sep-Oct;14(5): 38-50.

4. Siever D. Entraining tones and binaural beats, Mind Alive Inc.; 2009. [cited 2015 March 10]. Available from: http://mindalive.com/default/assets/Fil e/article\%2012.pdf.

5. Ivanov $P$, Amaral L, Goldberger A, et al. Multifractality in human heartbeat dynamics. Nature. 1999 Jun 3;399 (6735):461-5. doi: 10.1038/20924.

6. Gierałtowski J, Żebrowski J \& Baranowski R. Multiscale multifractal analysis of heart rate variability recordings with a large number of occurrences of arrhythmia. Phys Rev E Stat Nonlin Soft Matter Phys. 2012 Feb;85(2 Pt 1): 021915. doi: 10.1103/PhysRevE.85.0219 15.

7. Popivanov D, Stomonyakov V, Minchev Z, et al. Multifractality of decomposed EEG during imaginary and real visual-motor tracking. Biol Cybern. 2006 Feb; 94(2): 149-56. doi: 10.1007/ s00422-005-0037-5.

8. Dutta S, Ghosh D \& Chatterjee S. Multifractal detrended fluctuation anal- ysis of human gait diseases. Front Physiol. 2013; 4:274. doi: 10.3389/fphys. 2013.00274.

9. Casciaro F, Laterza V, Conte S, et al. Alpha-rhythm stimulation using brain entrainment enhances heart rate variability in subjects with reduced HRV. World Journal of Neuroscience. 2013;3: 213-20. doi: 10.4236/wjns.2013.34028.

10. Pitstick B. CES 2014 Wearable \& fitness tech trends: going mainstream. Technical Report of Moor Insights \& Strategy, 2014. [cited 2015 March 10]. Available from: http://goo.gl/WEL2mv.

11. Minchev Z. Cyber threats analysis in on-line social networks with a study on user response. IT4Sec Reports 115, [cited 2015 March 10]. doi: 10.11610/ it4sec.0115.

12. Niedermeyer E \& da Silva F. Electroencephalography. New York: Lippincott Williams \& Wilkins; 2005.

13. David Delight Plus Operators Manual, Mind Alive Inc., April 2014 [cited 2015 March 10]. Available from: http://goo.gl/s03ndz.

14. Mina M. Real time emotion detection using EEG, [Technical Report] The American University in Cairo, 2009, [cited 2015 March 10]. Available from: www.cse.aucegypt.edu/ rafea/CSCE590 /Spring09/Mina/Mina.pdf.

15. Minchev Z \& Gatev P. Psychophysiological evaluation of emotions due to the communication in social networks. Scripta Scientifica Medica, 2012; 44(1): 125-8. 